\title{
La sordoceguera en la educación actual: la educación física como apoyo a la inclusión.
}

Deaf Blindness in Today's Education: Physical education in support of inclusion. Taubblindheit in der Bildung heute: Sportunterricht als Unterstützung der Inklusion. La surdicécité dans l'éducation aujourd'hui : l'éducation physique comme support à l'inclusion.

A surdez na educação hoje: a educação física como suporte para a inclusão. La sordocecità nell'educazione oggi: l'educazione fisica come supporto all'inclusione.

Глухота в образовании сегодня: физическое воспитание как поддержка инклюзивности.

今日の教育における難聴：インクルージョンの支援としての体育 当今教育中的袭哑问题：体育是融入社会的支撑。

\author{
Carmen Flores Melero \\ Universidad de Jaén (España) \\ cfm00016@red.ujaen.es
}

Fecha recepción:18/09/2020

Páginas 129-135

Fecha aceptación:29/12/2020

\section{Resumen.}

La educación inclusiva se ha caracterizado en su plenitud por la capacidad de adaptar e incrementar la participación de aquellos alumnos y alumnas con necesidades educativas especiales de cualquier índole. Al mismo tiempo esta educación inclusiva ha sido apoyada por diferentes áreas del currículum educativo actual, más concretamente el área de Educación Física ha sido una de que se han reestructurado, su profesorado se ha formado y han logrado tener éxitos con los alumnos y alumnas con necesidades educativas especiales. Tanto es así, que en ocasiones son capaces de, a través de la observación, darse cuenta de las necesidades que pueda tener un alumno o alumna. En gran parte, la sordera en niños y niñas ha sido un impedimento en muchas áreas, sin embargo, estos niños y niñas dicen sentirse realmente bien al ir a la clase de Educación Física, asimismo sucede con la ceguera. En estudio revisamos la bibliografía sobre la sordoceguera y la experiencia de la Educación Física y Especial en este tipo de diversidad funcional.

Palabras clave: educación; aulas especiales; inclusión; sordoceguera; educación física

\section{Abstract.}

Inclusive education has been fully characterized by the ability to adapt and increase the participation of students with special educational needs of any kind. At the same time this inclusive education has been supported by different areas of the current 
educational curriculum, more specifically the area of Physical Education has been one of that have been restructured, their teachers have been trained and have been successful with pupils with special educational needs. So much so, that sometimes they are able to, through observation, realize the needs that a student may have. In large part, deafness in boys and girls has been an impediment in many areas, however, these boys and girls say they feel really good about going to the Physical Education class, as well as blindness. We reviewed the literature on deaf-blindness and the experience of Physical and Special Education in this type of functional diversity.

Keywords: education; special classrooms; inclusion; deaf-blindness; physical education

\section{1.-Introducción.}

Resulta necesaria la revisión de la literatura acerca de los docentes de educación física, así como de los docentes de educación especial que tratan en su día a día de fomentar y mejorar la calidad educativa de los alumnos y alumnas que tienen necesidades educativas especiales. Más concretamente a continuación vamos a conocer cuáles son las necesidades que se requieren en los centros ordinarios y públicos de nuestro país, así como cuales son las mejoras y los avances susceptibles de cambio para fomentar la inclusión y la educación del alumnado de nuestro país.

De igual modo y más concretamente vamos a conocer la realidad del alumnado con sordoceguera, adentrándonos en las inmensas grietas de esta complicada, especial y única diversidad funcional. Así pues, también se añadirán las necesidades de este tipo de alumnado en los colegios ordinarios, obteniendo por consecuencia la necesidad de formación del profesorado de educación especial y de educación física, quienes ya sabemos, poseen el gran papel de ayudar a mejorar la inclusión de este tipo de alumnado, tanto a nivel académico como social.

\section{2.-Marco Teórico.}

Este trabajo consiste en una revisión de la literatura, que se ha realizado gracias a la revisión bibliográfica de diversos documentos de sociedades científicas, entregadas al ámbito educativo y sanitario, además de aspectos relacionados con estos: educación inclusiva, educación especial, educación física, actividad física, inclusión, sordoceguera, evolución, entre otros.

Este marco teórico ha sido efectuado tras realizar una búsqueda de palabras clave en diferentes plataformas y bases de datos de alto impacto, en la plataforma de recursos de información ERIC, la búsqueda fue realizada tanto en español como en inglés. Se puso en práctica una búsqueda exhaustiva de documentos con una serie de criterios de inclusión y exclusión que veremos más adelante. Las bases de datos de alto impacto donde se localizó dicha información fueron: Scopus, MPDI, Scielo y Web of Science. Así mismo, para abarcar la mayor cantidad de información sobre los temas anteriormente expuestos, se hizo un análisis de las referencias bibliográficas 
que contenía cada uno de los documentos ya revisados y que habián resultado de interés.

La calidad de los estudios que se incluyen en esta revisión de la literatura es alta.

A continuación, se exponen los criterios de inclusión y exclusión: se incluyen artículos en cualquier idioma, se incluyen artículos independientemente de su localización, se incluyen aquellos que contengan en sus resúmenes información de interés relacionada con la educación inclusiva, la educación especial, la sordoceguera y la educación física, se incluyen estudios de cualquier año, pues en esta revisión se intenta contemplar lo más relevante de la educación inclusiva y especial a lo largo de los años. Por el contrario, se excluyen aquellos artículos, que no resulten de interés y que en sus resúmenes no se haga referencia a ninguno de los temas anteriormente expuestos.

\section{1.-La educación especial.}

En primer lugar, cabe destacar que las aulas especiales persiguen varios factores fundamentalmente, entre ellos está: Mejorar la respuesta educativa en contexto lo más normalizados posibles con alumnos y alumnas con necesidades educativas especiales graves y permanentes; del mismo modo, también persiguen el favorecer una respuesta educativa a los alumnos y alumnas que requieren de apoyos continuos y permanentes; así mismo también aumentar y promover las experiencias de atención a la diversidad en contextos lo más flexibles posibles; apostar por una mejor respuesta, ofreciendo oportunidades para aquellos alumnos y alumnas que lo requieran por sus necesidades educativas especiales, en especial aquellos con TEA y/o patologías afines, así como alumnado pluridiscapacitado; y finalmente aumentar y fomentar las actitudes respetuosas y de atención a la diversidad en toda la comunidad educativa y de la sociedad. (Abellán \& Currás, 2019).

En segundo lugar y haciendo referencia a García-Cairó (1980), ya explicaba y destacaba las críticas y las posibles alternativas de aprovechamiento y mejora de las aulas de educación especial en los colegios de nuestro país. Entre estas propuestas de mejora, García- Cairó destacaba la necesidad de una adecuada dotación de personal y medios para llevar a cabo la prevención de deficiencias y anomalías; por otro lado hacía referencia a que la plaza de docente especial fueran psicólogos, esto actualmente se ha ido perfeccionando y formando el papel del PT, sí siendo cierto que los psicólogos formen o deberían formar parte de la plantilla escolar y formar de manera colaborativa y conjunta con el resto del profesorado en las tareas y formulación de la pedagogía del centro; el trabajo de este referente psicólogo o PT escolar debe ser en primer lugar la observación y el análisis de los problemas conjuntos e individuales que plantean los alumnos y alumnas de manera individual o con el grupo clase. Siendo imprescindible conocer la realidad al completo de cada alumno o alumna en su medio natural y su entorno escolar, siendo este el aula de educación especial.

Haciendo también rotunda incidencia en lo que fue la Ley General de Educación (1970) ya derogada, que trataba el sistema educativo y a los centros docentes y 
resumiendo decía que la Educación Especial tendría como finalidad la de preparar, mediante el adecuado tratamiento educativo, a los deficientes e inadaptados para una incorporación a la vida social tan plena como sea posible.

Se puede considerar cómo poco a poco se ha ido dándole más relevancia a la Educación Especial y se ha mejorado la incidencia en este ámbito, así como la capacitación del personal docente, tanto de Pedagogía Terapéutica, como de Educación Física, que es el perfil docente que vamos a revisar a continuación.

\section{2.-La Educación Física para alumnado con Necesidades Educativas Especiales}

La Educación Especial, según Abarca, Clemente, \& García, (2013), presenta alumnado con diferentes tipos de diagnósticos, por lo que el docente de Educación Física, así como el mismo currículum ha de estar preparado para este tipo de alumnado, alumnado con: trastorno del espectro autista, parálisis cerebral, Síndrome de Down, enfermedades degenerativas, trastorno generalizado del desarrollo, entre otros muchos. Por lo tanto, el papel de la Educación física se fundamenta en la necesidad de implementar actividades y ejercicios físicos acordes a sus características y que a su vez, dichas actividades sean capaces de fomentar diferentes habilidades básicas o destrezas más complejas en el alumno o alumna. Se ha de partir de una programación adecuada a la ley educativa vigente en cada momento, aún sin haber gran información sobre la adaptación del currículum ordinadio de Educación Física. De igual manera, se observa una gran necesidad de propuestas metodológicas para conseguir objetivos dentro del aula de Educación Física para trabajar con este tipo de alumnado. El profesorado de Educación Física deberá trabajar y proponer actividades adaptadas a los elementes del currículum de sus programaciones, así como elaborar propuestas docentes adaptadas a las características de cada uno de sus alumnos y alumnas.

\section{3.-La sordoceguera}

Según la Federación Española de Sordoceguera (2020), la sordoceguera es una discapacidad única, que tiene característica y por supuesto, una serie de necesidades propias, que son: la pérdida de la capacidad visual y la capacidad auditiva. Esta discapacidad se trata de una condición específica, haciendo especial hincapié en que no es la suma de dos discapacidades. Tampoco es una enfermedad, aunque sí puede ser que una enfermedad pueda ser la causante de la sordoceguera.

Tabla 1. La Sordoceguera (Tipos, Causas y Consecuencias) 
Revista Internacional de Apoyo a la Inclusión, Logopedia, Sociedad y Multiculturalidad.

Volumen 7, Número 1, enero 2021, ISSN: 2387-0907. DOI: https://dx.doi.org/10.17561/riai.v7.n1.10

\begin{tabular}{|c|c|c|}
\hline $\begin{array}{l}\text { Sordoceguera } \\
\text { congénita }\end{array}$ & $\begin{array}{l}\text { Los nacimientos } \\
\text { prematuros, la rubeola, la } \\
\text { sífilis, toxoplasmosis y los } \\
\text { síndromes de Charge, } \\
\text { Cogan y Hurler. }\end{array}$ & $\begin{array}{l}\text { Imposibilidad de desarrollar el } \\
\text { lenguaje, dificultad en el aprendizaje } \\
\text { de conductas o modos de actuación, } \\
\text { incomprensión del mundo que le } \\
\text { rodea así como a los objetos que le } \\
\text { rodean. }\end{array}$ \\
\hline $\begin{array}{l}\text { Sordoceguera } \\
\text { adquirida }\end{array}$ & $\begin{array}{lr}\text { Anoxia, } & \text { tumores, } \\
\text { ictus, síndrome } & \text { de } \\
\text { Usher, síndrome } & \text { de } \\
\text { Wólfram } & y \\
\text { neurofibromatosis. } & \end{array}$ & $\begin{array}{l}\text { Comunicación alterada, dificultad en } \\
\text { el acceso a la información, dificultad } \\
\text { de conocer lo que está pasando y } \\
\text { por consecuencia para la toma de } \\
\text { decisiones, orientación y movilidad } \\
\text { es decir, lo relativo al uso del propio } \\
\text { cuerpo en relación con el entorno. }\end{array}$ \\
\hline
\end{tabular}

Fuente de elaboración propia

Ilustración 1. La sordoceguera (Momento de aparición y nivel de funcionamiento)

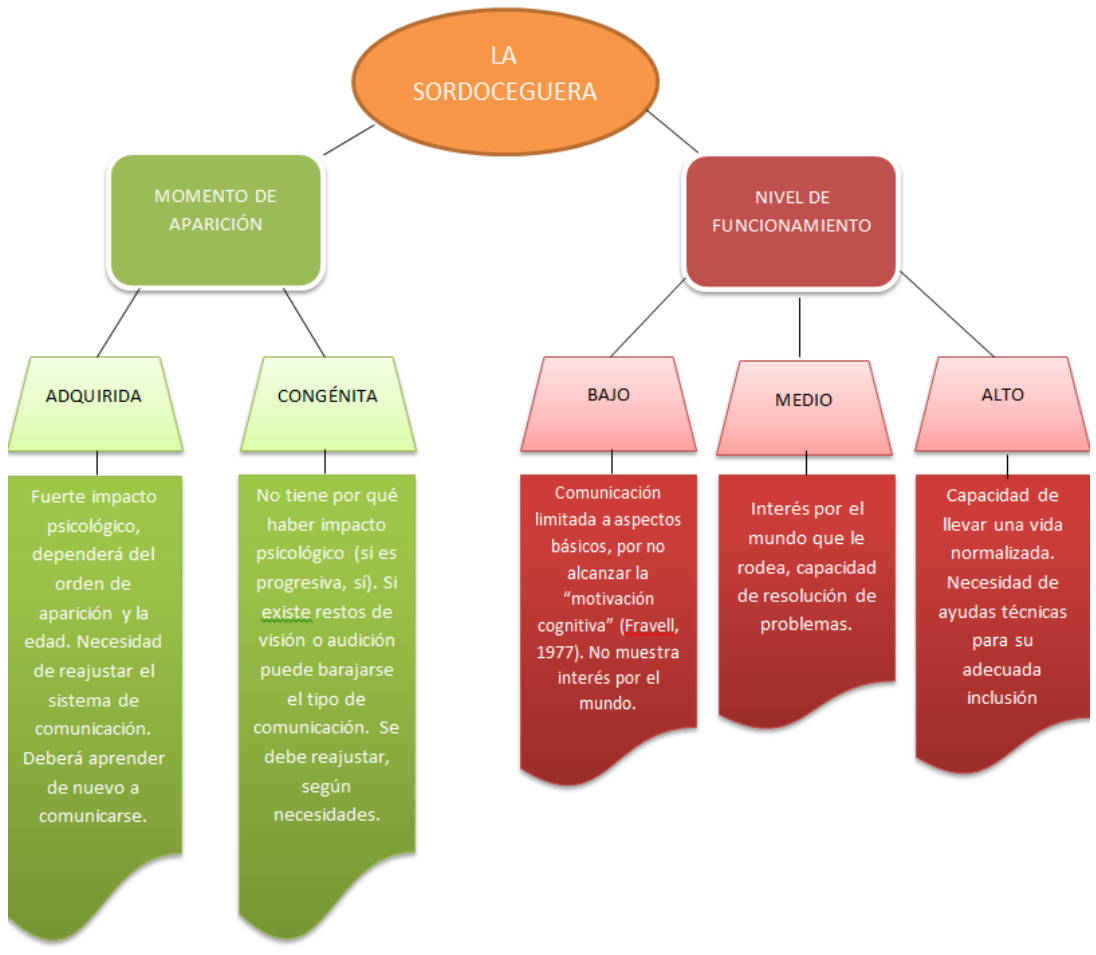

Fuente de elaboración propia. 


\section{5.-Conclusión.}

En primer lugar, se concluye que la Educación Especial y la Educación Física son una adecuada mezcla para lograr la inclusión educativa que en la actualidad tanto buscamos. En segundo lugar, se debe de conocer que los dos tipos de sordoceguera son muy diferentes, siempre habrá diferencias con cada alumno y alumna, pues es muy diferente haber conocido lo que es el mundo (sordoceguera adquirida), que el no saber ni lo que significa esta palabra (sordoceguera congénita). Con la persona con una sordoceguera adquirida habrá que intervenir mucho en el ámbito emocional, social, para que este tipo de personas no se rindan y continúen con la gran base de ventaja que les llevan a las personas con sordoceguera congénita, con estas últimas habrá de intentar que experimenten el mundo en todas sus vertientes y aprovechando todas sus posibilidades. El tipo de desarrollo de ambas va a ser totalmente diferente, pues la persona que es sordociega congénita, habrá desarrollado de manera sus otros sentidos, pero no habrá logrado llegar a conocer nunca el mundo en su inmensidad, no reconocerá lo que es relacionarse de manera extensa con su entorno, aunque habrá aprendido a vivir de esta manera, por otro lado, la persona con sordoceguera adquirida, habrá aprendido a vivir de una manera que va a tener que imaginar, vivir del recuerdo, superar el proceso de tener una vida "normal" (sin que me guste usar esta palabra), para pasar a vivir con una gran restricción, a nivel psicológico y desarrollo social y emocional serán muy diferentes, pues esta última habrá de necesitar mucho apoyo en este sentido. Finalmente, solo queda añadir que la formación de los docentes de educación física es muy importante, puesto que ellos ejercen un gran papel en la educación de niños y niñas con necesidades educativas especiales.

\section{6.-Discusión.}

Es relevante decir, que se ofrece la oportunidad de seguir investigando en este ámbito y crear nuevos hilos de discusión, en cuanto y en tanto a la sordoceguera y sus niveles como a la calidad de la formación docente universitario de los futuros docentes de pedagogía terapéutica y educación física, con el objetivo de mejorar la calidad de la enseñanza para el alumnado con necesidades educativas especiales y fomentar la inclusión de los mismos en los centros y aulas ordinarias de nuestro país.

\section{7.-Referencias.}

Abarca, A., Clemente, J.A.J. \& García, L. (2013). Adaptación del currículum ordinario de educación física en educación primaria y propuesta metodológica para alumnado escolarizado en centros de educación especial. Ágora para la Educación Física y el Deporte, 15(3), 228-242. https://dialnet.unirioja.es/servlet/articulo?codigo $=4530404$

Abellán, R. M., \& Currás, M. P. (2019). Special classrooms in Spain: Comparative analysis. Siglo Cero, 50(3), 89-120. https://doi.org/10.14201/scero201950389120 
Federación Española de Sordoceguera. (2020).¿Qué es la sordoceguera? Consultado el 9 de diciembre de 2020.

García Cairó, M. (1980). Crítica y alternativas al aprovechamiento de las aulas de educación especial en los colegios nacionales de E.G.B. Infancia y Aprendizaje, 3(10), 121-122. https://doi.org/10.1080/02103702.1980.10821799 